\title{
DISC BATTERY IMPACTION IN THE OESOPHAGUS
}

\author{
N. C.E. Okpala \\ ENT Department, St George's Hospital, Blackshaw Road, Tooting, \\ London SW17 0QT, UK.
}

\begin{abstract}
The recent growing popularity of electronic miniature toys has made disc battery ingestion increasingly common. The impaction of these miniature toys can result in a range of complications such as minor oesophageal alkali burns, tracheo-oesophageal and aorto-oesophageal fistula. A case report in which an eleven month old boy swallowed a foreign body-a disc battery is presented. Occasional retching was observed with no respiratory distress upon arrival at the Queen Alexandra Hospital, Portsmouth, United Kingdom. Even though he was irritable, systemic examination did not reveal any obvious abnormality. The slightly-eroded battery with surrounding black debris was eventually removed. On the chest radiograph, the battery looked similar to coins which are more commonly ingested and could be easily mistaken for coins with dire consequences. The chest radiograph revealed the battery as a circular radio-opaque shadow with a peripheral double rim. It is important for medical practitioners to recognise this fact and be able to identify a battery from a coin which has a similar shape but a single rim since the history of the foreign body swallowed may be as unclear as it was in this case.
\end{abstract}

Keywords: Disc battery, coin, oesophageal foreign body/ impaction, double-rim outline

\section{INTRODUCTION}

Disc battery ingestion is increasingly common with the growing popularity of electronic miniature toys (Litoritz, 1983; Anand et al., 2002). When impacted, disc batteries can cause complications, which range from minor oesophageal alkali burns to tracheo-oesophageal fistula and even aorto-oesophageal fistula (Tibballs et al., 2002). Accidental ingestion of a foreign body occurs frequently in children.
Coins are commonly ingested and they look similar to the disc battery on a chest radiograph. The only difference is the double-rim outline for the disc battery and a single rim for the coin on the radio-opaque shadow. The battery may therefore be easily mistaken for a coin. The management of these cases differ since impacted coins can be given time to pass with little or no morbidity. However, the same cannot be said of batteries, which contain corrosive substances and may cause 
necrosis of the mucosa in case of leakage (Arana et al., 2001; Volle et al., 1989). A case of disc battery ingestion is presented below.

\section{CASE REPORT}

An eleven month old boy swallowed a foreign body about three o'clock in the afternoon. The mother had no idea of the nature of the foreign body, however she felt it might have been a coin. The boy was playing with his toy box and suddenly appeared to be choking with traces of cyanosis. His mother patted him on the back and that seemed to have settled him for sometime. Then followed suddenly bouts of coughing and retching, but on arrival at the Queen Alexandra hospital, the child was not in respiratory distress though there continued to be occasional retching. On a careful examination, he was found to be irritable but not in any obvious distress. The temperature, respiratory rate and pulse were within normal limits. Systemic examination did not reveal any obvious abnormality. An X-ray of the neck and chest revealed a foreign body in the upper oesophagus. The object was flat and round and located at the level of the first rib (T1) (Figure). On a first look, it appeared like a coin. However on closer examination, it looked different from the typical coin seen on X-ray.

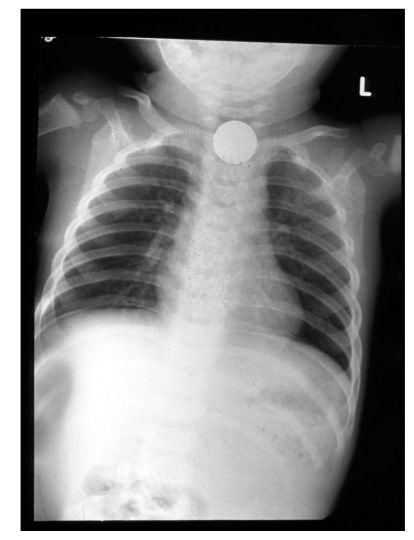

Fig. 1: Chest X-ray showing disc battery at the level of the first rib.
The boy was subsequently taken to the theatre three hours after the incident. Pharyngooesophagoscopy revealed a three-volt flat lithium battery just below the cricopharyngeus. The battery was surrounded by black debris, which was sucked out. The battery appeared to be slightly eroded. The oesophageal mucosa looked stained but it was intact. Post-operatively, the child was stable without any problem. He was discharged the following day.

\section{DISCUSSION}

Disc battery ingestion is increasingly encountered in medical practice. There is an increase in its percentage among ingested foreign bodies (Bothwell and Cable, 2003). Coin ingestion is still much common in young children with an incidence of up to $5 \%$ and the mean age just under 3 years (Litovitz and Schmitz, 1992). Coins are relatively frequently trapped in the oesophagus.

The mechanism of injury due to oesophageal impaction of disc batteries may be due to electrolyte leakage, alkali produced by external current (electrolysis), mercury toxicity and pressure necrosis (Tibballs et al., 2002). The most important factor leading to complications is the duration of impaction of foreign body. There are two factors, which influence the chance of impaction of the disc battery. First is the size of the battery and the other factor is the nature of the oesophagus (Arana et al., 2001; Volle et al., 1989; Bothwell and Cable, 2003). In the present study, the battery had been lodged for about three hours. There was no significant damage to the oesophageal mucosa probably because of the short duration. The larger the size, the more likely it is to get impacted. Batteries larger than $22 \mathrm{~mm}$ in diameter have more chances of impaction (Arana et al., 2001). However, the battery measured $20 \mathrm{~mm}$. It was lodged in the narrowest part of the oesophagus which is often susceptible to spasms. Weak passive peristalsis also makes the oesophagus susceptible.

The orientation of the battery in the oesophagus determines the nature of the injury. This was deduced from studies done in rabbits with lithium

2 Journal of Science and Technology, Vol. 28, No. 1, April 2008 
batteries (Volle et al., 1989). The voltage of the battery is retained and significant injury to the oesophagus occurs, whatever the orientation of the battery is. When the cathode faces the trachea, it too can be injured. With the above knowledge, disc batteries should be removed as soon as possible. In this study, the battery was lying transversely with the cathode facing the trachea. Tracheal injury has only been reported when the battery has been lodged for a long time. The orientation can only be properly deduced at oesophagoscopy. It did not influence management in this study. The orientation becomes an important factor when the battery has been lodged for a long time.

On the chest radiograph, the battery appears as a circular radio-opaque shadow with a double rim on the periphery (Figure 2). The chest radiograph needs to be adequately penetrated to feature the double rim outline clearly as was in this study.

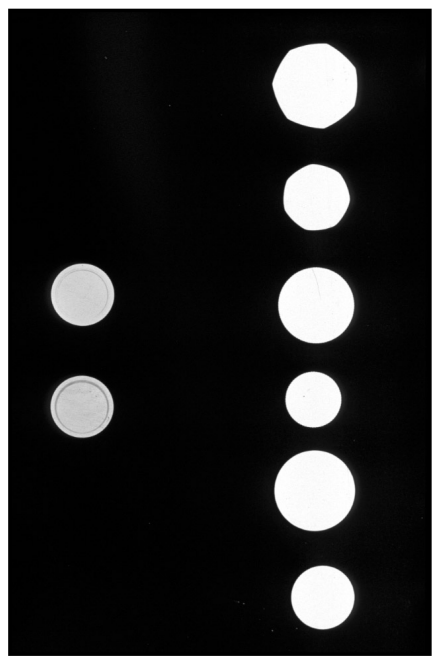

Fig. 2: X-ray showing disc batteries on the left and coins on the right for comparison.

Coins, which are more commonly ingested, look similar to the batteries on the radiograph and the latter can easily be mistaken for coins with dire consequences such as oesophageal burns, perfora- tion, stricture, mediastinitis, tracheo-oesophageal and aorto-oesophageal fistula. It is important to recognise this fact since the history of the type of foreign body may be unclear as it was in this study.

\section{ACKNOWLEDGEMENT}

Many thanks to all those who have helped in the writing of this paper especially my brother, Odilichukwu Okpala.

\section{REFERENCES}

Anand, T.S., Kumar, S., Wadhwa, V. and Dhawan, R. (2002). Rare case of spontaneous closure of tracheo-oesophageal fistula secondary to disc battery ingestion. International Journal of Paediatric Otorhinolaryngology. 63: 57-59.

Arana, A., Hauser, B., Hachimi-Idrissi, S. and Vandenplas, Y. (2001). Management of ingested foreign bodies in childhood and review of the literature. Eur J Pediatr. 160: 468-472.

Bothwell, D.N. and Cable, B.B. (2003). Chronic ingestion of a zinc based penny. Paediatrics. 111 (3): 689-691.

Litoritz, T.L. (1983). Button battery ingestions: a review of 56 cases. Journal of American Medical Association. 249: 2495-2500.

Litovitz T.L and Schmitz BF. (1992). Ingestion of cylindrical and button batteries: an analysis of 2382 cases. Paediatrics. 89: 747-757.

Tibballs, J., Wall, R., Velandy-Koottayi, S., Stokes, K.B., Cochrane, A., Barnes, R. and Kimber, C. (2002). Tracheo-oesophageal fistula caused by electrolysis of a button battery impacted in the oesophagus. Paediatr Child Health. 38: 201-203.

Volle, E., Beyer, P. and Kaufmann, H.J. (1989). Therapeutic approach to ingested button-type batteries. Magnetic removal of ingested button-type batteries. Pediatr Radiol. 19: 114118. 